\title{
Le substantif latin mundus: un avatar de l'étrusque mutna?
}

Jean-Paul Brachet

(Sorbonne University)

\section{The Latin Substantive mundus: a Borrowing from Etruscan mutna?}

\begin{abstract}
We try to demonstrate that the Latin substantive mundus is a loanword from Etruscan mutana/mutna. From a formal point of view, it is quite possible since a sequence ${ }^{*}$-tn- became -nd- at a very early stage in Latin. Mutna refers to a sort of a tomb or sarcophagus. As we know, lat. mundus can refer to an underground cavity (mundus Cereris). It is worth noting that Etruscan borrowed from Latin-Faliscan the noun cela /cella/ in order to refer also to an underground tomb or burial-place, which meaning is very close to the one of mutna. So we assume that Latin borrowed mutna from Etruscan and that mundus primitively referred to an underground cavity which was especially intended for supplies (which meaning was preserved in the locution in mundo esse).
\end{abstract}

\section{Keywords}

Latin; Etruscan; loanword; mundus; mutna; cella 
Le point de départ de cette étude nous est venu d'inscriptions funéraires étrusques dans lesquelles se rencontrent respectivement le nom proprement étrusque mutana/mutna et l'emprunt au falisque cela. ${ }^{1}$

\section{Étr. mutna et cela.}

Mutna «sarcophage, tombe» est un mot courant en étrusque: ${ }^{2}$

AT (Ager Tarquiniensis) 1.34:

eca : mutna : velOurus : sta<t>lanes larisaliśla

«ceci est le sarcophage de Velthur Statlanes, fils de Laris.»

Ta (Tarquinia ) 1.81 (200-100 av. J.-C.):

$\theta u i \cdot c l \theta i \cdot \operatorname{mutn}\{a i\} \theta i$

vel $\cdot$ veluaa $\cdot$ avils

cis $\cdot$ zaArmisc

seitit•ialiśa

«ici, dans ce sarcophage, (repose) Vel, fils de Vel, 23 ans, fils de Seitithi.»3

Cela «chambre mortuaire, tombe» est attesté une fois:

Ta 1.66 ( $c a 300$ av. J.-C.):

vel: aties : velOurus :

lemniśa : celati : cesu

«vel Aties, fils de Velthur et Lemni, (a été) enseveli dans cette chambre.» ${ }^{4}$

En étrusque, le terme générique qui désigne la tombe, la sépulture, paraît être $s u \theta i$ (śüi). Mutna, moins fréquent, doit être plus spécifique; on le rend habituellement par «sarcophage». Néanmoins, mutna reste un nom courant et bien attesté, notamment dans la formule récurrente eca mutna suivi du nom du défunt au génitif «ceci est la tombe/ le sarcophage de...»: ${ }^{5}$

Ta 1.28 : eca mutana cutus velus

«Ceci est la tombe de Vel Codonius.»

AT 1.36 : eca mutna ram $\theta$ as mania(l)

1 Équivalent de lat. cella - le falisque ne notant pas les géminées.

2 «La signification du mot mut(a)na ne pose aucun problème de compréhension. Ce mot se rencontre en effet dans une douzaine d'inscriptions de la région de Tarquinies, au nominatif accompagné du pronom démonstratif $(e) c a$, le groupe $(e) c a$ mut(a)na servant, à l'évidence, à désigner le support sur lequel l'inscription était apposée. Ce support étant systématiquement un sarcophage, on peut donc être raisonnablement certain que tel était le sens du mot.» Hadas-Lebel (2016: p. 66).

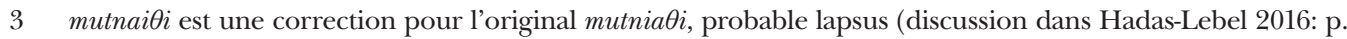
66). Pour l'interprétation de la forme casuelle, on se reportera à Hadas-Lebel (2016: pp. 66-67), qui fait des formes nominales pourvues de la postposition $-\theta i$-ti des locatifs «inessifs», indiquant le lieu où l'on est.

4 Hadas-Lebel (2016: p. 102) discute la forme celati: la postposition - $\theta i$ s'ajoute normalement au locatif en

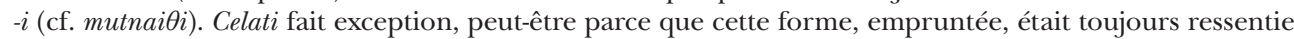
comme un xénisme par les locuteurs et, comme telle, non déclinée.

5 On se reportera aux Etruskische Texte de Meiser pour avoir les attestations. 
«Ceci est la tombe de Ramtha Mania.»

Le nom cela / cella/ est à peu près unanimement considéré comme un emprunt au falisque, bien que ce mot ne soit pas attesté dans la documentation falisque. Bakkum (2009: p. 181) écrit à ce sujet: «In my view, the material can be interpreted equally well, if not better, by assuming an original Latin-Faliscan Word cella with the meaning 'covered or enclosed room, chamber' (whether underground or above ground), which could easily be applied to underground burial chambers in areas where these were used. (Faliscan lectus and cubo are also everyday Latin words with a secondary funerary meaning.)» Toutefois, en latin, comme le rappelle Bakkum, cella ne n'applique jamais à une tombe; il est vrai que les usages funéraires des Romains, majoritairement incinérants, s'y prêtaient mal. Pourtant, les Romains plaçaient parfois les urnes dans des sortes de caveaux, qui regroupaient les restes d'une même famille, ou beaucoup plus; on avait alors affaire à un columbarium. ${ }^{6}$

C'est précisément le fait que le nom mutna s'applique à une certaine forme de tombe comportant une sorte de cuve ou de chambre qui nous incite à en rapprocher certains emplois de lat. mundus.

\section{Les expressions in mundo esse / habere.}

In mundo esse est une locution archaïque, qui disparaît des textes au cours $\mathrm{du}_{\mathrm{II}} \mathrm{I}^{\mathrm{e}} \mathrm{s}$ av. J.-C. Le sens de base de l'expression n'est pas douteux, il est confirmé par une glose de Festus: «être à portée de main, disponible». Les attestations sont assez peu nombreuses pour être toutes citées:

Pl. Cas. 563-565: stultitia magna est mea quidem sententia, hominem amatorem ullum ad forum procedere, in eum diem quoi quod amet in mundo siet.

«C'est grande sottise, à mon sens, quand on est amoureux, d'aller au forum, le jour précisément où l'on a à sa disposition l'objet de son amour.» (trad. Grimal, 1971).

Pl. Ep. 618: quippe ego quoi libertas in mundo sitast.

«J'ai mon affranchissement en poche» (trad. Ernout, 1935); «Sans doute parce que ma liberté est désormais assurée?» (trad. Grimal, 1971).

Symétriquement, in mundo habere signifie «avoir à sa disposition, à portée de main»:

Pl. Pers. 45: nempe habeo in mundo.

«L'affaire est dans le sac» (trad. Ernout, 1938a); «Alors, je l'ai déjà dans ma poche.» (trad. Grimal, 1971).

De «être à portée de main, disponible» in mundo esse glisse vers le sens de «attendre quelqu'un, être réservé à quelqu'un», la locution se restreignant souvent à l'expression d'un sort peu enviable (il s'agit certainement d'un emploi ironique au départ):

6 Cf. Cagnat \& Chapot (1916, ch. 16, Monuments funéraires: pp. 321-328). Il y avait aussi les vieilles tombes à puits, puticuli. 
Pl. Asin. 263-264:

certe hercle ego quantum ex augurio auspicioque intellego, aut mihi in mundo sunt uirgae aut atriensi Saureae.

«Par Hercule, autant que je puis comprendre l'augure et le présage, il y a des verges qui nous attendent, ou moi ou l'intendant Sauréa.» (trad. Grimal, 1971).

Pl. Asin. 315-316:

ergo mirabar quod dudum scapulae gestibant mihi,

hariolari quae occeperunt sibi esse in mundo malum.

«Voilà pourquoi je me demandais ce qui faisait que, depuis quelque temps, les épaules me démangeaient: elles ont commencé à annoncer qu'il y avait une raclée qui les attendait.» (trad. Grimal, 1971).

Pl. Pseud. 499-501: - - pistrinum in mundo scibam, si id faxem, mihi.

- non a me scibas pistrinum in mundo tibi,

cum ea mussitabas?

«Je savais que le moulin m'attendait, si je l'avais fait. - Et tu ne savais pas que de mon côté il t'attendait aussi, quand tu ne soufflais mot?»(trad. Ernout, 1938b).

Enn. Ann. 467-468 V: tibi uita / seu mors in mundo est.

«que ce soit la vie ou la mort qui t'attende».

Caecil. 276-277: profertoque nobis in mundo futurum / lectum.

«et apporte-nous un lit qui soit à notre disposition à l'avenir.»

Caecil. 278: namque [mihi] malum in mundost, ere.

«en effet, un malheur est sur nous, maître.»

À quel mundus faut-il rapporter in mundo esse? à l'adjectif mundus, - $a$, -um ou à un mundus substantif? Perplexité du rédacteur de l'article du TLL (Kamptz), p. 1632: «locutio a Plauto, Ennio, Caecilio ... usurpata qua ratione c. 1. mundus cohaereat, non satis perspicitur, quoniam neque ad notionem solitam neque ad usus peculiares 1. 64 p. 1631, 10 notatos quadrare uidetur; uix melius applicari potest ad 2.3.4. mundus.»

Un fragment de Lucilius, ${ }^{8}$ 519-520 Marx, doit certainement être mis en relation avec in mundo esse:

legauit quidam uxori mundum omne penumque.

quid mundum atque penum? quid non? quis diuidet istuc?

«Un homme légua à sa femme tout son trousseau et toutes les provisions de ménage; mais le trousseau et les provisions de ménage, qu'est-ce? que n'est-ce pas? Qui tranchera un tel débat?» (trad. Charpin, 1979: 16, 6, pp. 83-84).

Ce fragment témoigne de la difficulté que les Latins eux-mêmes éprouvaient parfois à comprendre mundus. La traduction par «trousseau» est suggérée par Aulu-Gelle, qui rapproche le mundus muliebris, mais rien ne garantit que ce soit la bonne. S'il s'agissait vraiment du mundus muliebris, il semble bizarre que le mari laisse à sa femme son propre trousseau. Ce qui nous paraît à noter ici, c'est que mundus est associé à penus, qui dé-

7 = 496-497 dans l'éd. Skutsch, Oxford, 1985.

8 Fragment transmis par Aulu-Gelle, parce que c'est la seule attestation d'un mundum neutre, 4, 1, 3: mundum quoque muliebrem Lucilius in satirarum XVI. non uirili genere, ut ceteri, sed neutro appellauit his uerbis... (le texte transmis par Aulu-Gelle diffère un peu de celui qu'a édité Charpin dans la C.U.F., 1979). 
signe les provisions, les réserves. ${ }^{9}$ Ne peut-on en inférer que mundus désigne dans le fragment de Lucilius une sorte de garde-manger, de lieu de stockage des réserves (penus), un cellier - en d'autres termes, la richesse de la maisonnée, dans une économie rurale? On ajoutera à l'appui de cette interprétation un passage du Stichus de Plaute, où la locution in mundo habere apparaît dans le contexte d'un banquet et signifie «avoir quelque chose en réserve» pour le servir à ses invités:

Stich. 476-478:

- Non edepol possum. - Quid grauare? censeas.

nescio quid uero habeo in mundo. - I modo,

alium conuiuam quaerito tibi in hunc diem.

«- Pourquoi te faire prier? Accepte. J'ai un je ne sais quoi à te servir. - Va donc; cherchetoi un autre convive pour aujourd'hui.» (trad. Ernout, 1938b).

Nous avons un autre indice chez Plaute que la locution était encore analysable à son époque, dans un passage qui contient un jeu de mots sur in marsuppio et in mundo:

Poen. 782-783: $\quad$ - $\quad$ idque in istoc adeo aurum inest marsuppio.

uae uostrae aetati! - id quidem in mundo est tuae.

«Et précisément cet or est dans la bourse que tu portes. - Malheur à vous! - C'est ton malheur à toi qui est déjà là!» (trad. Grimal, 1971).

Les philippes sont certes dans la poche de Lycus, mais les ennuis sont dans son mundus et l'attendent.

Nous faisons l'hypothèse que in mundo esse est une expression de la vie domestique signifiant «être dans la réserve, dans le garde-manger», c'est-à-dire «être à portée de main, disponible». Cette hypothèse trouve un appui dans la glose de Festus, p. 97 L: in mundo dicebant antiqui, cum aliquid in promptu esse uolebant intellegi.

L'équivalence faite par Festus entre in mundo esse et in promptu esse est d'autant plus intéressante que la famille de promptus, promere, promus, désigne le fait de tirer les provisions du cellier. La locution in mundo esse provient donc des usages domestiques d'un domaine rural. Un signe du sens matériel qu'avait l'expression à l'origine est la variante in mundo situm esse, que nous avons rencontrée en Pl. Ep. 618.

\section{Mundus, cave à provisions, et mundus Cereris.}

Mundus a donc probablement désigné dans le latin très archaïque, antérieur à notre documentation, une sorte de cave à provisions, de cellier. Le mundus Cereris est assurément la plus belle survivance de ce mundus «cave à provisions» («cave à grain»). Le mot subissait alors la concurrence, qui s'est avérée victorieuse, de cella. Ce dernier substantif désigne notoirement une cave où l'on range des provisions, un cellier; c'est un mot très fréquent chez Caton et les agronomes:

9 Des rapprochements étymologiques sont possibles pour penus: sk. panasá- (m. ou n.) "fruit de l'arbre à pain”, cf. Mayrhofer (1963: s.v.) et lit. pẽnas "nourriture, fourrage”, penéti "nourrir, engraisser", penýba "engraissement, entretien", cf. Fraenckel (1962 : s.v.). 
Cat. Agr. 3, 2: patrem familiae uillam rusticam bene aedificatam habere expedit, cellam oleariam, uinariam, dolia multa.

«Il convient que le père de famille ait un domaine à la campagne bien construit, une cave pour l'huile, une pour le vin, de nombreuses jarres.»

Cat. Agr. 13, 2: in cellam oleariam haec opus sunt: dolia olearia, opercula...

«Pour la cave à huile, les choses suivantes sont nécessaires: des jarres à huile, des couvercles, ...»

Cat. Agr. 65, 2: quam calidissimum torcularium et cellam habeto.

«Il faut maintenir le pressoir et la cave le plus chauds possible.»

Colum. 1, 6, 54: pars autem fructuaria diuiditur in cellam oleariam, torculariam, cellam uinariam, defrutariam.

«Les bâtiments à provisions se divisent en une cave à huile, un pressoir, une cave à vin, un cellier pour le moût.»

Colum. 1, 6, 56: torcularia praecipue cellaeque oleariae calidae esse debent.

«Les pressoirs particulièrement, ainsi que les caves à huile, doivent être chauds.»

Si les domaines de mundus et de cella se recouvrent, c'est parce que les choses désignées ont une similitude de forme: il s'agit d'une sorte de cuve ou caveau, pour employer un mot français qui convient aussi aux usages funéraires. Or le mundus primitif, paraît avoir été un dépôt de fondation, une sorte de caveau souterrain, si l'on en croit Plutarque:

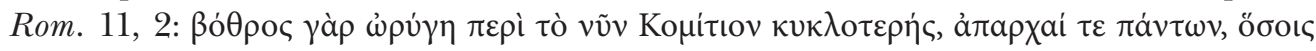

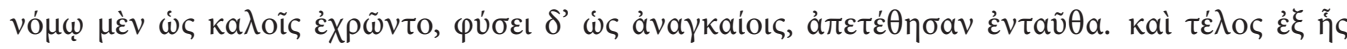
ả

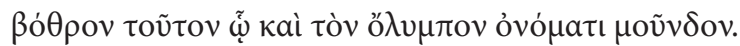

«Une fosse fut creusée du côté de ce qui est aujourd'hui le Comitium, de forme circulaire, et ils y déposèrent les prémices de toutes les choses dont ils usaient en tant qu'elles étaient bonnes selon la loi, et en tant qu'elles étaient nécessaires selon la nature. Et à la fin, chacun apporta une poignée de la terre du pays d'où il était venu, ils jetèrent les poignées de terre dans le même endroit et les mélangèrent. Ils appellent ce fossé de la même façon que la voûte céleste: mundus.»

Certains ont pensé depuis longtemps que le mundus Cereris était primitivement une sorte de silo à grains: "Nous rattachons donc les rites du mundus au culte le plus ancien qui nous soit accessible de Cérès "indigène". Ce rituel a bien pu être bivalent: rien ne nous oblige à rejeter complètement l'interprétation agraire de W. Fowler, qui malgré le silence des textes présente une certaine vraisemblance générale. Elle n’est nullement incompatible avec le culte des Mânes: c'est un fait bien connu que les divinités du sol peuvent être à la fois infernales et agraires. Le culte que Cérès recevait au mundus présentait probablement ce double caractère.» ${ }^{10}$ L'idée d'un mundus silo à grains était déjà en effet exprimée par W. Warde Fowler: «I conjecture that it was the place in which was stored, not, or not only the grain of the last harvest which would be needed for food, and for which the storehouse (penus) would need to be frequently opened in the old farmhouse,

10 Le Bonniec (1958: p. 184). 
but the place of safety which the seed-corn was stored.» ${ }^{11}$ Plus loin: «The mundus as Cato describes it, though on the Palatine in his day it would be only a symbolic survival from the original storing-place, seems to me strongly to suggest a use for human beings as well as ghosts ... We may imagine it [le mundus] as a kind of cellar with an opening in the centre of its roof.» ${ }^{12}$ De fait, lorsqu'il était ouvert, le mundus souterrain mettait le monde des hommes en communication avec les êtres infernaux:

Macr. Sat. 1, 16, 16-18: cum mundus patet, nefas est proelium sumere. (...) Inchoari bellum decebat nec Saturni festo, qui sine ullo tumultu bellico creditur imperasse, nec patente mundo, quod sacrum Diti patri et Proserpinae dicatum est, meliusque occlusa Plutonis fauce eundum ad proelium putauerunt. Vnde et Varro ita scribit: mundus cum patet, deorum tristium atque inferum quasi ianua patet.

«Quand le mundus est ouvert, il n'est pas permis d'entreprendre une guerre. Il n'était pas convenable de commencer une guerre lors de la fête de Saturne, dont on croit qu'il gouverna sans connaître le moindre tumulte guerrier, ni lorsque le mundus était ouvert, moment sacré voué à Dispater et à Proserpine - et on estima qu'il était préférable d'aller au combat lorsque la gueule de Pluton est fermée. De là ce qu'écrit Varron: quand le mundus est ouvert, c'est pour ainsi dire la porte des dieux du malheur et des enfers qui est ouverte.»

\section{Relation entre le «mundus d'en haut» (voûte céleste) et le «mundus d'en bası.}

Pour les Latins eux-mêmes, c'était en vertu de leur similitude de forme que la fosse à provisions et la voûte céleste étaient désignées par le même nom mundus: ${ }^{13}$

Fest. p. 144-146 L: sic refert Cato in commentariis iuris ciuilis: mundo nomen impositum est ab eo mundo qui supra nos est: forma enim eius est, ut ex is qui intrauere cognoscere potui, adsimilis illae.

«Caton rapporte ceci dans ses commentaires sur le droit civil: le mundus [d'en bas] a reçu son nom du mundus qui est au-dessus de nous: en effet, d'après ce que j'ai pu apprendre de ceux qui y sont entrés, sa forme est assez semblable à celle de l'autre.»

Le mundus était une fosse circulaire, nous dit Plutarque. ${ }^{14}$ L'habitation humaine primitive est une imago mundi. Elle fut le plus souvent une simple fosse arrondie, généralement à demi enterrée. Au plan symbolique, la forme circulaire exprime notoirement la clôture,

11 Warde Fowler, W. (1920). Mundus patet. In Idem, Roman Essays and Interpretations (pp. 24-39). Oxford: Clarendon Press, pp. 26-27; cité par Le Bonniec (1958: pp. 179-180).

12 Ibid. p. 27.

13 Synthèse sur les emplois astronomiques de mundus dans Le Bœuffle (1987, s.v. mundus, $\mathrm{n}^{\circ}$ 808, pp. 187-191). Le Bœuffle constate qu'«il est difficile de déterminer les aires sémantiques de caelum et de mundus», mundus, absent chez Plaute et Térence, paraissant plus savant, caelum plus usuel. À partir du sens de

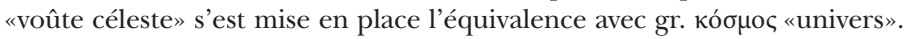

14 Pour la structure du mundus, cf. Basanoff (1942: pp. 4-7). 
la totalité. L'apparition de la maison rectangulaire, qui peut être découpée en pièces et être agrandie, est le signe de changements de mentalité fondamentaux..$^{15}$ On peut dès lors penser que mundus a désigné au départ une fosse voûtée, ce qui lui aurait permis de s'appliquer aussi bien à un cellier de stockage de denrées qu'à la voûte céleste. Le sens de «voûte, cavité» doit se retrouver dans la seule attestation de mundus qu'on ait chez Ennius, et qui laisse entendre que mundus a désigné au départ un objet distinct de caelum: Scipio (varia 9-12 V, p. 214): mundus caeli uastus constitit silentio et Neptunus saeuus undis asperis pausam dedit, sol equis iter repressit ungulis uolantibus, constitere amnes perennes, arbores uento uacant.

«La vaste cavité du ciel s'arrêta en silence, Neptune le déchaîné ordonna une pause aux flots violents, le soleil freina ses chevaux aux sabots volants, les fleuves qui ne cessent de couler s'arrêtèrent, le vent cessa d'agiter les arbres.»

A priori, le génitif caeli peut s'interpréter comme génitif d'appartenance («le mundus qui appartient au caelum») ou de définition («le mundus qu'est le caelum»). Cette dernière interprétation paraissant peu probable, on suivra Le Bœuffle qui suggère de prendre mundus au sens de «cavité». ${ }^{16}$

Du point de vue idéologique, mundus reflète une vision du monde d'agriculteurs sédentaires: centre de l'habitation, en tant que réserve à grain et à provisions, mundus est aussi centre du monde, axis mundi. ${ }^{17}$ "Le nomade chasseur-cueilleur saisit la surface de son territoire à travers ses trajets; l'agriculteur sédentaire construit le monde en cercles concentriques autour de son grenier», écrit A. Leroi-Gourhan..$^{18}$ Il arrive même que le grenier devienne le symbole de l'univers maîtrisable. ${ }^{19}$ On comprend dès lors pourquoi le mundus s'ouvrait notamment le 24 août, veille des Opiconsiuia, et pouvait servir à prédire l'état de la récolte à venir. ${ }^{20}$ Ce centre du monde faisait communiquer avec les dieux chtoniens, qui sont au premier chef les divinités agraires. ${ }^{21} \mathrm{R}$. Caillois avait noté à propos du mundus des Latins: «À Rome, à dates fixes, on soulève la pierre qui ferme le mundus: c'est un trou du Palatin qu'on tient pour la voie d'accès au monde infernal, pour la contraction de ce monde lui-même, et, comme son nom l'indique, pour le pen-

15 Voir à ce sujet Cauvin (1997: pp. 175-180): «L'habitat au carré».

16 Le Bouffle (1987: p. 187).

17 Cf. Briquel (1993: pp. 95 sq.) pour le mundus comme axe du monde.

18 Leroi-Gourhan (1965, ch. XIII, Les symboles de la société: p. 157).

19 Ibid. p. 168.

20 Cf. Scolie de Berne in Verg. Buc. 3, 104-105: aput antiquos fuit altissimus puteus, in quem descendebat puer, quo cognosceret anni prouentus «du temps des Anciens existait un puits très profond, dans lequel descendait un enfant afin de s'informer de la récolte de l'année», cité par A. Magdelain (1976: p. 109).

21 De manière générale, l'ouverture des récipients de stockage alimentaire donne souvent accès à l'autre monde et permet aux âmes de s'échapper. L'ouverture des jarres à vin, la $\pi \bullet$ oryía des Anthestéries athéniennes a parfois été comprise en ce sens (que les Anthestéries soient une fête des morts, la formule

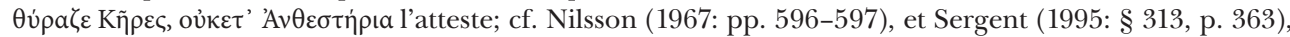
qui cite également un parallèle chez les Hittites, qui associaient l'ouverture des jarres alimentaires, au printemps, à la célébration des rois morts). 
dant exact du monde des vivants dont il est le symétrique dans l'au-delà. Il représente à la fois le résumé du Grand Espace en face de l'étendue profane et l'orifice qui permet leur communication.» ${ }^{22}$

\section{Lat. mundus, emprunt à étr. mutna?}

Puisque, en étrusque, mutna et cela servent à désigner un type de sépulture consistant en une sorte de cuve, chambre ou caveau, et, que, en latin, certains emplois très anciens de mundus laissent supposer un sens comparable de cave, caveau (le contexte funéraire en moins), proche de celui de cella (terme lui aussi exclu du contexte funéraire en latin), on peut se demander si mundus ne pourrait pas être un emprunt à mutna. Si cela est, en étrusque, un emprunt certain au falisque, mundus, symétriquement, pourrait très bien être l'avatar de mutna en latin. Comme on sait, le substantif mundus - sans parler pour le moment de l'adjectif mundus, - $a$, - um - n'a toujours pas reçu d'étymologie satisfaisante. ${ }^{23}$ Lancé au XIX ${ }^{\mathrm{e}}$ siècle, le rapprochement, douteux sur le plan formel, ${ }^{24}$ avec le nom étrusque connu sous les variantes $\operatorname{mun} \theta u \chi, \operatorname{mun} \theta \chi, \operatorname{mun} \theta u$ apporte peu du point de vue du contenu; ${ }^{25}$ il s'agirait du nom d'une déesse. En un mot, c'est expliquer obscura per obscuriora. Parmi les rapprochements formellement possibles, mutna reçoit, nous semble-t-il, un appui intéressant du côté du sens. Du point de vue formel, mundus peut provenir de étr. mutana - mutna avec syncope -, si l'emprunt est assez ancien. La question principale est celle du traitement de *-tn- et *-tVn- en latin. Cette question, débattue, est évoquée par Meiser (1997: §85, pp. 121-122). De même que *- $d n$ - aboutit normalement à $-n d-{ }^{26}$ de même une séquence *-tVn- ou*-tn-devient généralement - $n d$-. L'exemple qui paraît le plus net, celui auquel recourt Meiser, est le verbe pandō,-ere, qui remonte à *patane/o-. Le verbe d'état («essif») correspondant est pateō, ère. La racine, bien connue, se termine par une laryngale: ${ }^{*}$ peth $_{2}$. On peut discuter de la présence d'une voyelle d'anaptyxe en proto-latin: *patane/o- ou *patne/o-? La présence d'une anaptyxe $a$ est suggérée par osq. patensins < *patane-sēent «panderent». Si mundus est bien l'avatar de mutna, l'emprunt est alors extrêmement ancien, ${ }^{27}$ puisqu'il implique le traitement ${ }_{-}^{*} t V n-/ *_{-}-t n->-n d-$.

22 Caillois (1950: p. 150).

23 Voir p. ex. notre article de 2007. S.v. mundus «toilette, parure de la femme», Ernout \& Meillet (2001) note qu' «il semble qu'il y ait eu deux (ou trois) mots différents, un adjectif mundus et un substantif mundus "parure" et "monde" ".

24 Que serait devenue en latin la fin de ce mot étrusque, et que penser de la correspondance entre lat. $d$ et étrusque $\theta$ ?

25 Hypothèse évoquée sans autre commentaire dans Ernout \& Meillet (2001) s.v. mundus «cieux». De la discussion présente dans Breyer (1993: pp. 364-367), il ne ressort pas grand chose de net. L'auteure s'appuie surtout sur l'article de Pfiffig (1962), qui affirmait que mundus est un emprunt à mun $\theta$, et qui

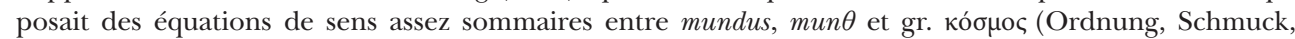
geordneter Platz).

26 C'est le type unda $<* u d-n \bar{a}$ (sur racine *wed-/*ud-, cf. v̋ $\delta \omega \rho$, water, etc.).

27 Nous ne nous hasarderons pas à avancer une date absolue. L'emprunt peut être aussi ancien que les premiers contacts entre Étrusques et Latins. 


\section{L'adjectif mundus, - $a$, -um.}

Quant à l'adjectif mundus, c'est un homonyme probable. D'aucuns lui ont trouvé un pedigree indo-européen acceptable, encore que non exempt de toute difficulté..$^{28}$ Ainsi

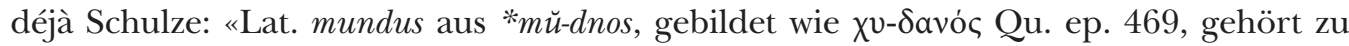
der durch sl. myti und ai. mútram bezeugten Wurzel $m \bar{u}$ "waschen" ebd. 170, bedeutet also ursprünglich "gewaschen" ». ${ }^{29}$ L'analyse a été affinée ensuite par Szemerényi 1950 et Puhvel 1976, qui posent *mūt-no- ${ }^{30}$ Pour le sens, cette étymologie est satisfaisante, l'idée de base de mundus étant bien celle d'une propreté obtenue par lavage:

P.-Fest. p. 127 L: mundus quoque appellatur lautus et purus.

«mundus signifie également nettoyé et propre»

Hor. Epist. 1, 5, 7: iamdudum splendet focus et tibi munda supellex.

«Déjà t'attendent, resplendissants, le foyer et de la vaisselle bien propre.»

Colum. 7, 3, 8: sint (stabula) mundissima.

«Qu'on maintienne les étables en parfait état de propreté.»

Colum. 7, 9, 14: mundissimum cubile desiderat.

«Le porc réclame une couche parfaitement propre.»

L'idée de propreté se retrouve dans le dérivé munditiēs (munditia):

Pl. Stich. 347-349: $\quad$ munditias uolo fieri. Ecferte huc scopas simulque harundinem, ut operam omnem araneorum perdam et texturam improbem deiciamque eorum omnis telas.

«Je veux qu'on fasse le ménage. Apportez les balais et aussi la tête de loup, que je détruise le travail des araignées, que je déchire leurs tissages et que je fasse descendre leurs toiles.» (trad. Grimal, 1971).

Sen. Epist. 86, 10: hoc quoque nobilissimi aediles fungebantur officio intrandi ea loca quae populum receptabant exigendique munditias et utilem ac salubrem temperaturam.

«Les plus remarquables de nos édiles s'acquittaient également de cette tâche d'entrer dans les lieux qui accueillaient le public et de vérifier la propreté et si la température était correcte et bonne pour la santé.»

Si l'on refuse ces tentatives étymologiques, on peut encore, comme Ernout l'avait fait,

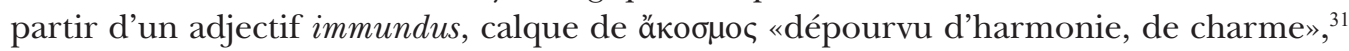
d'où aurait été tiré secondairement un adjectif positif mundus.

28 La notice de De Vaan (2008) s.v. mundus «clean, elegant » apporte peu. Le rapprochement avec des formes issues de la racine *meud- «se réjouir» (sk. múd-, móda- «joie, plaisir» entre autres) est peu probable pour des raisons sémantiques.

29 Schulze (1913) = Schulze (1933: p. 471).

30 Celui-ci fait de mundus une formation en *tno- sur la racine *mū- $\left({ }^{*} m u-H-\right)$ «baigner, laver». Quant à la racine de v.sl. myjo, myti, etc., on doit la retrouver encore en lat. dans mustum, autre mot dont la formation n'est pas limpide. Autre rattachement proposé dans $L I V$ s.v. *myewh ${ }_{1}^{-}$«(se) mouvoir».

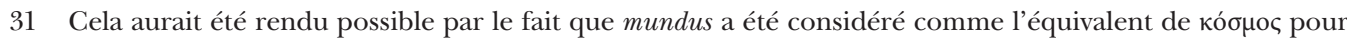
la désignation de l'«univers». 
L'origine du substantif mundus que nous venons de proposer ne prétend pas faire disparaître toutes les difficultés que soulève un tel mot. En tout cas, elle nous paraît possible et soutenable sur les plans formel et sémantique.

\section{Bibliographie}

Bakkum, G. C. (2009). The Latin Dialect of the Ager Faliscan. Amsterdam: University Press.

Basanoff, V. (1942). Les dieux des Romains. Paris: Presses universitaires de France.

Brachet, J.-P. (2007). Les différents mundus du latin: homonymie et histoire des mots. In A. Blanc, \& A. Christol (Eds.), L'homonymie dans les lexiques latin et grec (Association pour la Diffusion de la Recherche sur l'Antiquité; Nancy; Études anciennes, 33; pp. 47-62). Paris: Diffusion de Boccard. Breyer, G. (1993). Etruskisches Sprachgut im Lateinischen unter Ausschluss des spezifisch onomastischen Bereiches. Leuven: Peeters.

Briquel, D. (1993). Les Étrusques peuple de la différence. Paris: A. Colin.

Cagnat, R., \& Chapot, V. (1916). Manuel d'archéologie romaine, 1: Les monuments. Décoration des monuments. Sculpture. Paris: Picard.

Caillois, R. (1950). L’homme et le sacré (2éd.). Paris: Gallimard-Folio.

Cauvin, J. (1997). Naissance des divinités. Naissance de l'agriculture. La révolution des symboles au néolithique. Paris: CNRS Éditions.

Charpin, F. (Transl.). (1979). Gaius Lucilius: Satires (Collection des Universités de France, 2). Paris: Les Belles Lettres.

De Vaan, M. (2008). Etymological Dictionary of Latin and the other Italic Languages. Leiden: Brill.

Ernout, A. (Transl.). (1935). Plaute: Cistellaria, Curculio, Epidicus (Collection des Universités de France, 3). Paris: Les Belles Lettres.

Ernout, A. (Transl.). (1938a). Plaute: Mostellaria, Persa, Poenulus (Collection des Universités de France, 5). Paris: Les Belles Lettres.

Ernout, A. (Transl.). (1938b). Plaute: Pseudolus, Rudens, Stichus (Collection des Universités de France, 6). Paris: Les Belles Lettres.

Ernout, A., \& Meillet, A. (Eds.). (2001). Dictionnaire étymologique de la langue latine (retirage de la 4. éd. augm. d'additions et de corr.). Paris: Klincksieck.

Fraenckel, E. (1962). Litauisches etymologisches Wörterbuch (Vol. 1). Heidelberg: Winter.

Grimal, P. (Transl.). (1971). Plaute. Térence. Euvres complètes (Bibliothèque de la Pléiade, 224). [Paris]: Gallimard.

Hadas-Lebel, J. (2016). Les cas locaux en étrusque (Biblioteca di “Studi Etruschi”, 56). Roma: Giorgio Bretschneider editore.

Le Bøuffle, A. (1987). Astronomie, astrologie. Lexique latin. Paris: Picard.

Le Bonniec, A. (1958). Le culte de Cérès à Rome des origines à la fin de la République. Paris: Klincksieck.

Leroi-Gourhan, A. (1965). Le geste et la parole, II: La mémoire et les rythmes. Paris: Albin Michel.

$L I V=$ Rix, H. (2001). Lexicon der indogermanischen Verben. Die Wurzeln und ihre Primärstammbildungen ( $2^{\mathrm{e}}$ éd.). Wiesbaden: Reichert.

Magdelain, A. (1976). Le pomerium archaïque et le mundus. Revue des Études Latines, 54, 71-109. 
Mayrhofer, M. (1963). Kurzgefaßtes etymologisches Wörterbuch des Altindischen (Vol. 2). Heidelberg: Winter Universitätsverlag.

Meiser, G. (2014). Etruskische Texte. Editio minor. Teil 1: Einleitung, Konkordanz, Indices. Teil 2: Texte. Hamburg: Baar-Verlag.

Nilsson, M. P. (1967). Geschichte der griechischen Religion (Vol. I, $3^{\mathrm{e}}$ éd.). München: Beck.

Pfiffig, A. J. (1962). Etruskisches I: Zu lateinisch mundus und etruskisch mun . Die Sprache, 8, 142-145.

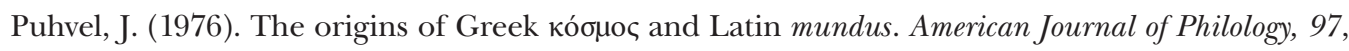
154-167.

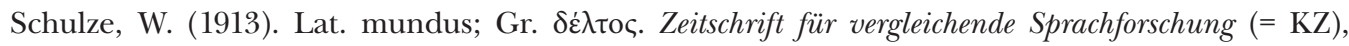
45(3), 235.

Schulze, W. (1933). Kleine Schriften. Zum 70. Geburtstag am 15. Dez. 1933 hrsg. vom Indogermanischen Seminar der Universität Berlin. Göttingen: Vandenhoeck \& Ruprecht.

Sergent, B. (1995). Les Indo-Européens. Paris: Payot \& Rivages.

Szemerényi, O. (1987). The Latin gerundive and other -nd-formations. Scripta minora, 2, 591-601 (= Transactions of the Philological Society, 1950, 169-179).

Jean-Paul Brachet, maître de conférences / brachet_jean-paul@orange.fr

Institut de Latin

Sorbonne Université, Faculté des Lettres

1, rue Victor Cousin, 75005 Paris, France 